\title{
New advances in ambient information systems
}

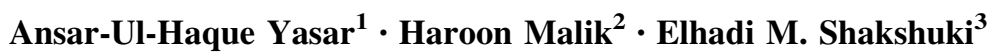

Published online: 3 March 2016

(c) Springer-Verlag Berlin Heidelberg 2016

This special issue is based on the best papers from the Sixth International Conference on Ambient Systems, Networks and Technologies (ANT), which was held at London, United Kingdom, on 2-5 June 2015. The conference attracted a large number of scientific papers that contributed to the state-of-the-art in the areas of pervasive and ambient information systems. All the papers selected for this special issue have been extended from their original versions and underwent three rounds of rigorous peer-review process. Based on the reviewers' feedback, as well as the evaluations of the Guest Editors, 8 papers were selected for this special issue from 12 invited submissions. The accepted papers cover interesting works on new developments in ambient and networked systems such as internetof-things (IoT), vehicular networks, context-aware computing, wireless sensors network (WSN), smart spaces, systems security and intelligent mobility.

The first paper by Negash et al. is entitled "LISA 2.0: Lightweight Internet of Things Service Bus Architecture Using Node Centric Networking". The authors introduce a lightweight, distributed, and embedded service bus called

Elhadi M. Shakshuki

elhadi.shakshuki@acadiau.ca

Ansar-Ul-Haque Yasar

ansar.yasar@uhasselt.be

Haroon Malik

malikh@marshall.edu

1 Transportation Research Institute, Hasselt University, Hasselt, Belgium

2 Division of Computer Science, Marshall University, Huntington, WV, USA

3 Jodrey School of Computer Science, Acadia University, Wolfville, Canada
LISA which follows a Node Centric Networking architecture. LISA is designed to provide interoperability for resource-constrained devices in IoT. It also enables a natural way of embracing the new IoT requirements, such as mobility and intermittent availability, through Node Centric Networking. LISA provides a simple application programming interface for developers, hiding the variations in platform, protocol or physical network, thus facilitating interoperability in IoT systems. LISA is inspired by Network on Terminal Architecture (NoTA), a service centric open architecture originated by Nokia Research Center. Extensive experimental results show the efficiency and scalability of LISA in providing a lightweight interoperability for IoT systems.

The second paper by Feukeu et al. is entitled "Doppler Shift Mitigation in a VANET using an Improved Direct Deviation Method (IDDM) approach". This paper proposes a solution to mitigate the effect of Doppler Shift (DS) affecting the Vehicular Ad hoc Networks (VANET) environment. Based on the Direct Derivation Method (DDM) derived from the principle of communication theory, an IDDM concept, analysis and design is developed and explored. Several critical tests are performed and it was demonstrated that not only the IDDM response exhibits the Bit Error Rate (BER) threshold limit described by the IEEE 802.11 standard; the approach offers for the same SNR value, a throughput improvement of up to $66.66 \%$ and an improved BER response of more than $25 \%$ compared to the DDM and Constant (Cte). A performance analysis also demonstrated that not only IDDM performs faster, it equally offers considerable improvement in term of efficiency over the DDM approach.

The third paper by Cassales et al. "Improving the Performance of Apache Hadoop on Pervasive Environments through Context-Aware Scheduling" proposes to improve 
Apache Hadoop scheduling through a context-aware approach. Apache Hadoop is the most popular implementation of the MapReduce paradigm for distributed computing, but its design does not adapt automatically to computing nodes' context and capabilities. By introducing context-awareness into Hadoop, the authors intent to dynamically adapt its scheduling to the execution environment. This is a necessary feature in the context of pervasive grids, which are heterogeneous, dynamic and shared environments. The solution has been incorporated into Hadoop and assessed through controlled experiments. The experiments demonstrate that context-awareness provides comparative performance gains, especially when some of the resources disappear during execution.

The fourth paper by Sheltami et al. is entitled "Wireless Sensor Networks for Leak Detection in Pipelines: A Survey". The authors of this paper provide a survey of recent methods of detecting pipeline leaks with special focus on software based methods. These methods include negative pressure wave, mass/volume balance, pressure point analysis, real time transient modeling, statistical methods as well as methods that employing digital signal processing. This paper also surveys some of the recent research attempts that focus on the employment of wireless sensor networks for leak detection and present research challenges that can be encountered in such environments.

The fifth paper by Bernardos et al. "Design and deployment of contactless hand-shape identification system for smart spaces" explores the feasibility of a contactless identification system based on hand features. The identification solution has been designed to be integrated with smart space applications and relies on a commercial 3D sensor (i.e. Leap Motion) for palm features capture. The first part of the paper is devoted to evaluate the significance of the different hand features and the performance of a set of classification algorithms. To build a testing dataset, 21 users contributed to it; for each user, the morphology of each hand has been gathered from 52 features, which include bones length and width, palm characteristics and relative distances among fingers, palm center and wrist. In order to get consistent samples and guarantee the best performance for Leap Motion device, the data collection system provides sweet spot control. This functionality guides the user to situate the hand in the best position and orientation with respect to the device. The selected classification strategies-nearest neighbor $(\mathrm{NN})$, supported vector machine (SVM), multilayer perceptron, logistic regression and tree algorithms - have been evaluated on Weka. We have found that relative hand-pose distances are more significant than pure morphological features. On this feature set, the highest Correct Classified Instances (CCI) rate is reached through the multilayer perceptron algorithm (>96\%), although all the evaluated classifiers provide a
CCI rate above $90 \%$. The analysis also gather how these algorithms perform with a variable number of users in the database, and what the sensitivity to the number of training samples is for each algorithm. Results show that there are different alternatives that are accurate enough for noncritical, immediate response applications. The second part of the paper focuses on the implementation of application examples that are integrated with a real-time hand-based identification system using NN. In particular, the applications enable customization and gesture-based control of the smart space. A five-user study has provided insight into the system performance and user experience. The results confirm the viability of using in-air hand shape recognition in smart space settings, although also it is still needed to deal with aspects that hinder faster and more accurate recognition.

The sixth by Fazili et al. "The Effect of Adopting Green SLA on Key Parameters of Optical WDM Networks" present Green Service Level Agreement (GSLA) awareness for the hybrid and traditional routing mechanisms by proposing a mathematical model for the amount of route greenness, and proposing two algorithms for the adoption of GSLA. The effect of the adoption of GSLA on a network that on average has green energy available for longer duration of a day is modeled by defining a one-step two-bytwo Markov matrix and representing the transformation through a state machine. This work also examines a reprovisioning algorithm to deal with the effect of re-provisioning of the established light paths in case of a change in the topology of a network. To study the effect of adopting GSLA, two scenarios with different arrival rates of connections and four performance parameters such as the average emission per lambda, average connection length, GSLA satisfaction and success rate are defined and analyzed over NSFnet. Results reveal that adopting the GSLA by routing mechanisms decreases the resource efficiency with both light and heavy traffic in return for less reduction in emission as compared to green and hybrid routing mechanisms.

The seventh paper by Maciejewski et al. is entitled "Large-scale microscopic simulation of taxi services. Berlin and Barcelona case studies". The authors of this paper present research on large-scale microscopic simulation of taxi services in Berlin and Barcelona based on floating car data collected by local taxi fleets. Firstly, Berlin's and Barcelona's taxi markets are shortly described and the demand and supply data obtained from FCD analyzed. Secondly, the online taxi dispatching problem formulation for this specific case is given, followed by the definition of two real-time rule-based heuristics used to dispatch taxis dynamically within the simulation. Finally, the simulation setup in MATSim is described, and the results obtained with both heuristics are analysed and 
compared in terms of dispatching performance, proving the effectiveness of the second strategy at different demand and supply scales.

The eight and the last paper by Cich et al. is entitled "Threshold settings for TRIP/STOP Detection in GPS Traces". The authors of this paper present two methods to extract stops and trips from GPS traces: the first one focuses on periods of non-movement (stops) and the second one tries to identify the longest periods of movement (trips). A stop corresponds to a location where the individual halts with the intention to perform an activity. In order to assert the quality of both methods, the results are compared to cases where the stops and trips are known by other means. First a set of traces was used for which the stops were identified by the traveler by means of a visual tool aimed at the alignment of manually reported periods in the diary to automatically recorded GPS coordinates. Second, a set of synthetic traces was used. Several quality indicators are presented; they have been evaluated using sensitivity analysis in order to determine the optimal values for the detector's configuration settings. Person traces (as opposed to car traces) were used. Individual specific behavior seems to have a large effect on the optimal values for threshold settings used in both the TRIP and STOP detector algorithms. Accurate detection of stops and trips in GPS traces is vital to prompted recall surveys because those surveys can extend over several weeks. Inaccurate stop detection requires frequent corrections by the respondent and can cause them to quit.

The guest editors would like to take this opportunity to thank all the authors for the efforts they put in the preparation of their manuscripts and for their valuable contributions. We wish to express our deepest gratitude to the referees who provided very useful and thoughtful feedback to our authors. Our sincere thanks go to the Editor-in-Chief for his kind help and support. We sincerely hope that you would enjoy reading this special issue. 